\title{
Cytokine release syndrome in severe COVID-19
}

\author{
By John B. Moore' ${ }^{1}$ and Carl H. June ${ }^{2}$ \\ ${ }^{1}$ Department of Hematology-Oncology, Walter Reed National Military Medical Center, Bethesda, MD, USA. ${ }^{2}$ Center for Cellular Immunotherapies, University of \\ Pennsylvania, Philadelphia, PA, USA. Email: john.b.moore74.mil@mail.mil; cjune@upenn.edu
}

\section{Lessons from arthritis and cell therapy in cancer patients point to therapy for severe disease}

In December 2019, a new strain of coronavirus, severe acute respiratory syndrome-coronavirus 2 (SARS-CoV-2), was recognized to have emerged in Wuhan, China. Along with SARS-CoV and Middle East respiratory syndromecoronavirus (MERS-CoV), SARS-CoV-2 is the third coronavirus to cause severe respiratory illness in humans, called coronavirus disease 2019 (COVID-19). This was recognized as a pandemic by the World Health Organization (WHO) in March 2020 and has had considerable global economic and health impacts. Although the situation is rapidly evolving, severe disease manifested by fever and pneumonia, leading to acute respiratory distress syndrome (ARDS), has been described in up to $20 \%$ of COVID-19 cases. This is reminiscent of cytokine release syndrome (CRS)-induced ARDS and secondary hemophagocytic lymphohistiocytosis (sHLH) observed in patients with SARS-CoV and MERS-CoV as well as in leukemia patients receiving engineered $\mathrm{T}$ cell therapy. Given this experience, urgently needed therapeutics based on suppressing CRS, such as tocilizumab, have entered clinical trials to treat COVID-19.

SARS-CoV-2 is a betacoronavirus that is most closely related to SARS-CoV. Both viruses use the angiotensinconverting enzyme-related carboxypeptidase (ACE2) receptor to gain entry to cells. This receptor is widely expressed in cardiopulmonary tissues but also in some hematopoietic cells, including monocytes and macrophages. A key feature of COVID-19 infection is lymphopenia (low blood lymphocyte count), which correlates with clinical severity (1). SARSCoV efficiently infects primary human monocytes and dendritic cells, whereas MERS-CoV infects monocytes and $\mathrm{T}$ cells via dipeptidyl peptidase 4 (DPP4) $(2,3)$. It is possible that SARS-CoV-2 also infects dendritic cells. T cell apoptosis and exhaustion resulting from defective activation due to dendritic cell dysfunction might contribute to the immunopathology of COVID-19 (2, 4). However, lymphopenia as a biomarker of poor prognosis for COVID-19 is not specific because it was also a biomarker that correlated with fatality in the 2009 influenza A (H1N1) pandemic (5).

CRS was found to be the major cause of morbidity in patients infected with SARS-CoV and MERS-CoV (6). Elevated serum concentrations of the cytokine interleukin-6 (IL-6) and other inflammatory cytokines are hallmarks of severe MERS-CoV infections (7). CRS is common in patients with COVID-19, and elevated serum IL-6 correlates with respiratory failure, ARDS, and adverse clinical outcomes $(8,9)$. Elevated serum C-reactive protein (CRP), a protein whose expression is driven by IL-6, is also a biomarker of severe betacoronavirus infection.

Betacoronavirus infection of monocytes, macrophages, and dendritic cells results in their activation and secretion of IL- 6 and other inflammatory cytokines. IL- 6 has prominent proinflammatory properties (see the figure). IL-6 can signal through two main pathways referred to as classic cis signaling or trans signaling (10). In cis signaling, IL-6 binds to membrane-bound IL-6 receptor (mIL-6R) in a complex with gp130; downstream signal transduction is mediated by JAKs (Janus kinases) and STAT3 (signal transducer and activator of transcription 3). Membrane-bound gp130 is ubiquitously expressed, whereas mIL-6R expression is restricted largely to immune cells. Activation of cis signaling results in pleiotropic effects on the acquired immune system ( $\mathrm{B}$ and $\mathrm{T}$ cells) as well as the innate immune system [neutrophils, macrophages, and natural killer (NK) cells], which can contribute to CRS (10).

In trans signaling, high circulating concentrations of IL6 bind to the soluble form of IL-6R (sIL-6R), forming a complex with a gp130 dimer on potentially all cell surfaces. The resultant IL-6-SIL-6R-JAK-STAT3 signaling is then activated in cells that do not express mIL-6R, such as endothelial cells. This results in a systemic "cytokine storm" involving secretion of vascular endothelial growth factor (VEGF), monocyte chemoattractant protein-1 (MCP-1), IL-8, and additional IL-6, as well as reduced E-cadherin expression on endothelial cells (11). VEGF and reduced E-cadherin expression contribute to vascular permeability and leakage, which participate in the pathophysiology of hypotension and pulmonary dysfunction in ARDS.

sHLH is a hyperinflammatory syndrome characterized 
by CRS, cytopenias (low blood cell counts), and multiorgan failure (including the liver) (12). In adults, sHLH is most commonly triggered by severe viral infections but also occurs in leukemia patients receiving engineered $\mathrm{T}$ cell therapy. In addition to elevated serum cytokines, high concentrations of ferritin are characteristic of sHLH. CD163expressing macrophages are implicated as the source of ferritin given their role in reticuloendothelial iron signaling, hence sHLH is alternatively known as macrophage activation syndrome. A retrospective study of COVID-19 patients found that elevated serum ferritin and IL-6 correlated with nonsurvivors (9).

Patients receiving chimeric antigen receptor (CAR) $\mathrm{T}$ cell therapy can also develop CRS and sHLH. This therapy involves engineering patient $\mathrm{T}$ cells to express CAR molecules that recognize antigens on tumor cells. When transplanted back into the patient, these engineered $\mathrm{T}$ cells target tumor cells, thereby activating immune clearance. Emily Whitehead, the first patient to receive CD19-targeted CAR T cells to treat pediatric B cell acute lymphoblastic leukemia in 2012, developed severe CRS and sHLH, leading to ARDS with multiorgan failure and hypotension that was refractory to standard treatment with steroids (13). Because of greatly elevated serum IL-6 in this patient, she was treated empirically with tocilizumab, an IL-6R antagonist approved at the time to treat rheumatic conditions such as juvenile idiopathic arthritis. She received a single dose of tocilizumab on day 7 after CAR $\mathrm{T}$ cell administration, with rapid resolution of fever within hours followed by weaning from vasopressors (which treat hypotension) and from ventilator support as ARDS resolved. Tocilizumab is now approved by the U.S. Food and Drug Administration (FDA) for the treatment of CAR T cell-induced CRS, with confirmed efficacy and minimal side effects in hundreds of patients.

The efficacy of IL-6-IL-6R antagonists for the treatment of CRS as well as SHLH underscores the central role of IL-6 signaling in the pathophysiology of cytokine-driven hyperinflammatory syndromes (10). Severe COVID-19 cases may benefit from IL-6 pathway inhibition given the associated CRS- and sHLH-like serum cytokine elevations. Indeed, preliminary results from an open-label study in 21 patients with COVID-19 treated with tocilizumab in China are encouraging (14). Fever subsided in all patients within the first day of receiving tocilizumab. Oxygen requirements were reduced in $75 \%$ of the patients (14).

Controlled clinical trials are under way worldwide to test IL-6 and IL-6R antagonists for the management of COVID-19 patients with severe respiratory complications. One issue to resolve is whether there will be differential effectiveness between IL-6 antagonists and IL-6R antagonists. Relevant to this is that IL-6R inhibitors can suppress both cis and trans signaling as well as trans presentation, a re- cently described third mode of signaling. Trans presentation involves IL-6 binding to mIL-6R expressed on an immune cell, which forms a complex with gp130 on $\mathrm{T}$ helper $17\left(\mathrm{~T}_{\mathrm{H}} 17\right)$ cells, leading to downstream $\mathrm{T}$ cell signaling that may be involved in ARDS $(10,11,15)$. However, IL-6 inhibitors can suppress only cis and trans signaling. The immediate goal of IL-6 antagonism is to ameliorate severe COVID-19 cases so that requirements for advanced care are minimized. The long-term goal should include a focus on the development of antivirals and vaccines that prevent or ameliorate the infection.

There are a number of caveats to consider, given the global urgency of mitigating the COVID-19 pandemic. In sepsis-associated ARDS, corticosteroids are often administered. However, corticosteroid use in SARS and MERS patients did not improve mortality and resulted in delayed viral clearance (6). As a result, the expert consensus from infectious disease authorities and the WHO is to avoid systemic corticosteroids in COVID-19 patients at present. A theoretical possibility is that the suppression of inflammation by IL- 6 antagonism might delay viral clearance. However, IL-6 blockade also results in rapid reduction of serum IL-10, an immunosuppressive cytokine secreted by macrophages, which may mitigate concerns about prolonging viral clearance (11). Moreover, one or two doses of an IL-6 antagonist are unlikely to result in complications, such as fungal infections or osteonecrosis of the jaw, which occur in patients dosed monthly on these drugs for chronic conditions such as rheumatoid arthritis. It is notable that tocilizumab was first approved for rheumatic conditions, then for CRS in patients receiving CAR T cell therapy, and is now being further repurposed for the COVID-19 pandemic. It is possible that IL- 6 directed therapies will be used in future pandemics involving other viruses such as influenza and Ebola (5, 11).

\section{REFERENCES AND NOTES}

1. X. Yang, Y. Yu, J. Xu, H. Shu, J. Xia, H. Liu, Y. Wu, L. Zhang, Z. Yu, M. Fang, T. Yu, Y. Wang, S. Pan, X. Zou, S. Yuan, Y. Shang, Clinical course and outcomes of critically ill patients with SARS-CoV-2 pneumonia in Wuhan, China: A singlecentered, retrospective, observational study. Lancet Respir. Med. 10.1016/S2213-2600(20)30079-5 (2020). doi:10.1016/S2213-2600(20)30079$\underline{5}$

2. H. Chu, J. Zhou, B. H.-Y. Wong, C. Li, J. F.-W. Chan, Z.-S. Cheng, D. Yang, D. Wang, A. C.-Y. Lee, C. Li, M.-L. Yeung, J.-P. Cai, I. H.-Y. Chan, W.-K. Ho, K. K.-W. To, B.-J. Zheng, Y. Yao, C. Qin, K.-Y. Yuen, Middle East Respiratory Syndrome Coronavirus Efficiently Infects Human Primary T Lymphocytes and Activates the Extrinsic and Intrinsic Apoptosis Pathways. J. Infect. Dis. 213, 904-914 (2016). doi:10.1093/infdis/jiv380 Medline

3. H. K. Law, C. Y. Cheung, H. Y. Ng, S. F. Sia, Y. O. Chan, W. Luk, J. M. Nicholls, J. S. M. Peiris, Y. L. Lau, Chemokine up-regulation in SARS-coronavirus-infected, monocyte-derived human dendritic cells. Blood 106, 2366-2374 (2005). doi:10.1182/blood-2004-10-4166 Medline

4. M. Zheng, Y. Gao, G. Wang, G. Song, S. Liu, D. Sun, Y. Xu, Z. Tian, Functional 
exhaustion of antiviral lymphocytes in COVID-19 patients. Cell. Mol. Immunol. 10.1038/s41423-020-0402-2 (2020). doi:10.1038/s41423-020-0402-2 Medline

5. R. Perez-Padilla, D. de la Rosa-Zamboni, S. Ponce de Leon, M. Hernandez, F. Quiñones-Falconi, E. Bautista, A. Ramirez-Venegas, J. Rojas-Serrano, C. E. Ormsby, A. Corrales, A. Higuera, E. Mondragon, J. A. Cordova-Villalobos; INER Working Group on Influenza, Pneumonia and respiratory failure from swineorigin influenza A (H1N1) in Mexico. N. Engl. J. Med. 361, 680-689 (2009). doi:10.1056/NEJMoa0904252 Medline

6. R. Channappanavar, S. Perlman, Pathogenic human coronavirus infections: Causes and consequences of cytokine storm and immunopathology. Semin. Immunopathol. 39, 529-539 (2017). doi:10.1007/s00281-017-0629-x Medline

7. A. R. Fehr, R. Channappanavar, S. Perlman, Middle East Respiratory Syndrome: Emergence of a Pathogenic Human Coronavirus. Annu. Rev. Med. 68, 387-399 (2017). doi:10.1146/annurev-med-051215-031152 Medline

8. G. Chen, D. Wu, W. Guo, Y. Cao, D. Huang, H. Wang, T. Wang, X. Zhang, H. Chen, H. Yu, X. Zhang, M. Zhang, S. Wu, J. Song, T. Chen, M. Han, S. Li, X. Luo, J. Zhao, Q. Ning, Clinical and immunological features of severe and moderate coronavirus disease 2019. J. Clin. Invest. 137244 (2020). doi:10.1172/ JCl137244 Medline

9. Q. Ruan, K. Yang, W. Wang, L. Jiang, J. Song, Clinical predictors of mortality due to COVID-19 based on an analysis of data of 150 patients from Wuhan, China. Intensive Care Med. 10.1007/s00134-020-05991-x (2020). doi:10.1007/s00134020-05991-x Medline

10. S. Kang, T. Tanaka, M. Narazaki, T. Kishimoto, Targeting Interleukin-6 Signaling in Clinic. Immunity 50, 1007-1023 (2019). doi:10.1016/j.immuni.2019.03.026 Medline

11. T. Tanaka, M. Narazaki, T. Kishimoto, Immunotherapeutic implications of IL-6 blockade for cytokine storm. Immunotherapy 8, 959-970 (2016). doi:10.2217/imt-2016-0020 Medline

12. C. B. Crayne, S. Albeituni, K. E. Nichols, R. Q. Cron, The Immunology of Macrophage Activation Syndrome. Front. Immunol. 10, 119 (2019). doi:10.3389/fimmu.2019.00119 Medline

13. S. A. Grupp, M. Kalos, D. Barrett, R. Aplenc, D. L. Porter, S. R. Rheingold, D. T. Teachey, A. Chew, B. Hauck, J. F. Wright, M. C. Milone, B. L. Levine, C. H. June, Chimeric antigen receptor-modified T cells for acute lymphoid leukemia. N. Engl. J. Med. 368, 1509-1518 (2013). doi:10.1056/NEJMoa1215134 Medline

14. X. Xu et al., Effective Treatment of Severe COVID-19 Patients with Tocilizumab. ChinaXiV 202003 (5 March 2020).

15. S. Heink, N. Yogev, C. Garbers, M. Herwerth, L. Aly, C. Gasperi, V. Husterer, A. L. Croxford, K. Möller-Hackbarth, H. S. Bartsch, K. Sotlar, S. Krebs, T. Regen, H. Blum, B. Hemmer, T. Misgeld, T. F. Wunderlich, J. Hidalgo, M. Oukka, S. RoseJohn, M. Schmidt-Supprian, A. Waisman, T. Korn, Trans-presentation of IL-6 by dendritic cells is required for the priming of pathogenic $T_{H} 17$ cells. Nat. Immunol. 18, 74-85 (2017). doi:10.1038/ni.3632 Medline

\section{ACKNOWLEDGMENTS}

This work was funded by NIH grant 2R01CA120409 (C.H.J.) and a sponsored research grant from the Parker Institute for Cancer Immunotherapy. J.B.M. is an employee of the U.S. Government. This work was prepared as part of his official duties. The views expressed in this article are those of the authors and do not necessarily reflect the official policy or position of the Department of the Navy, the Department of Defense, or the U.S. Government. The therapeutics discussed in this Perspective are undergoing clinical testing and are not currently approved for the treatment of COVID-19.

Published online 17 April 2020

10.1126/science.abb8925 


\section{Pathways leading to cytokine release syndrome}

Coronavirus infection results in monocyte, macrophage, and dendritic cell activation. IL-6 release then instigates an amplification cascade that results in cis signaling with $\mathrm{T}_{\mathrm{H}} 17$ differentiation, among other lymphocytic changes, and trans signaling in many cell types, such as endothelial cells. The resulting increased systemic cytokine production contributes to the pathophysiology of severe COVID-19, including hypotension and acute respiratory distress syndrome (ARDS), which might be treated with IL-6 antagonists such as tocilizumab, sarilumab, and siltuximab.

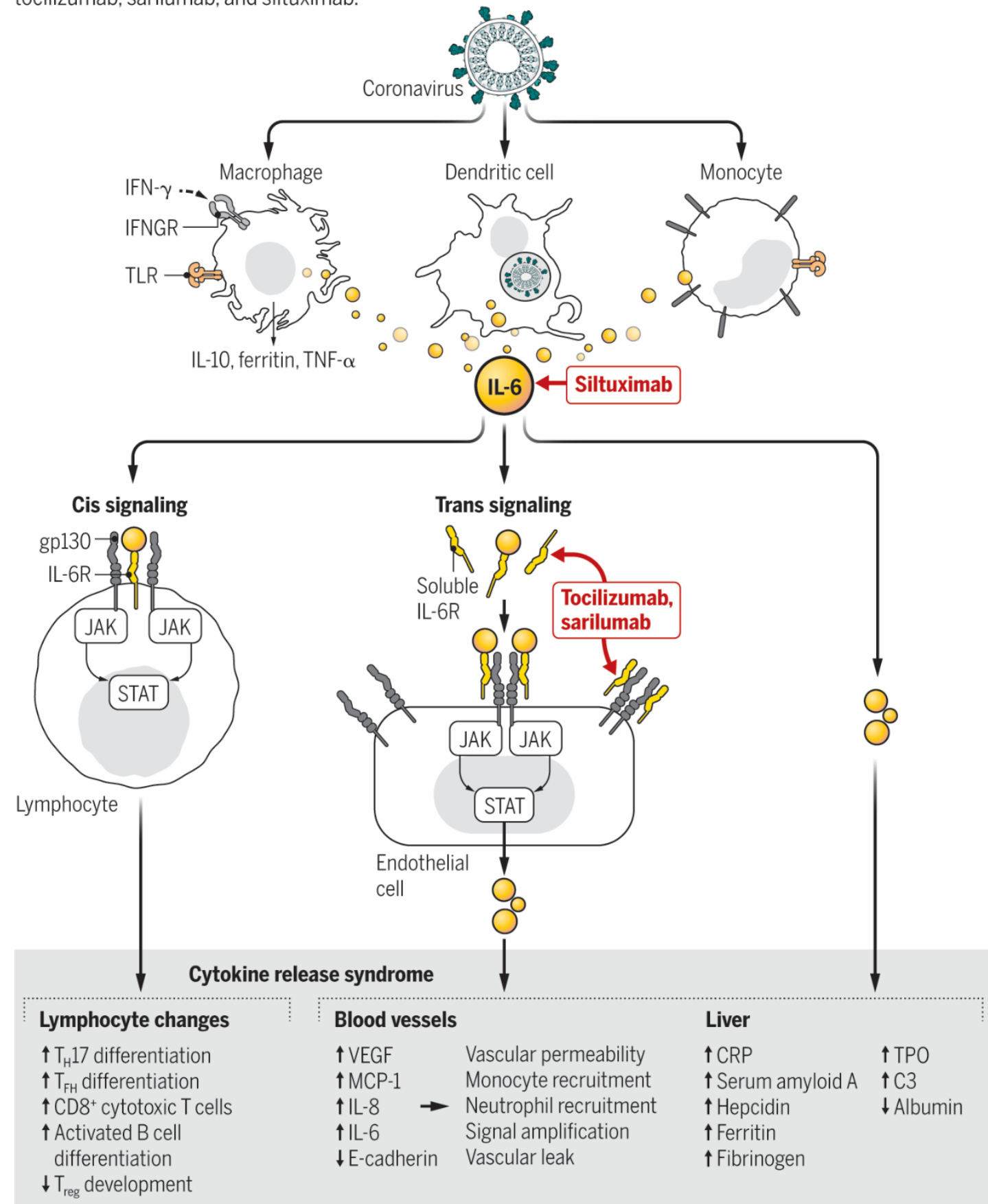

C3, complement 3; CRP, C reactive protein; IFN- $\gamma$, interferon- $\gamma$; IFNGR, IFN- $\gamma$ receptor; IL, interleukin; IL-6R, IL-6 receptor; JAK, Janus kinase; MCP-1, monocyte chemoattractant protein-1; STAT3, signal transducer and activator of transcription $3 ; T_{F H}$, $T$ follicular helper cell; $T_{H} 17, T$ helper 17 cell; TNF- $\alpha$, tumor necrosis factor- $\alpha$; TLR, Toll-like receptor; TPO, thrombopoietin; $\mathrm{T}_{\text {reg, }}$ T regulatory cell; VEGF, vascular endothelial growth factor. 